\title{
ON THE ORDER-THEORETICAL FOUNDATION OF A THEORY OF QUASICOMPACTLY GENERATED SPACES WITHOUT SEPARATION AXIOM
}

\author{
KARL H. HOFMANN and JIMMIE D. LAWSON
}

(Received 24 December 1981)

Communicated by J. B. Miller

\begin{abstract}
A Hausdorff space $X$ is said to be compactly generated (a $k$-space) if and only if the open subsets $U$ of $X$ are precisely those subsets for which $K \cap U$ is open in $K$ for all compact subsets of $K$ of $X$. We interpret this property as a duality property of the lattice $O(X)$ of open sets of $X$. This view point allows the introduction of the concept of being quasicompactly generated for an arbitrary sober space $X$. The methods involve the duality theory of up-complete semilattices, and certain inverse limit constructions. In the process, we verify that the new concept agrees with the classical one on Hausdorff spaces.
\end{abstract}

1980 Mathematics subject classification (Amer. Math. Soc.): 06 A 12, 06 A 15, 54 D 50.

Keywords and phrases: $k$-space; compactly generated space, duality, semilattice, continuous lattice.

A topological Hausdorff space $X$ is said to be a compactly generated space, or simply a $k$-space if and only if the compact subsets $K \subseteq X$ determine the topology $O(X)$ of $X$ in the sense that a subset $U$ of $X$ is open exactly if $U \cap K$ is open in $K$ for all compact subsets $K$ of $X$. A slightly weaker form of Hausdorff separation, called "axiom $t_{2}$ ", has turned out to be the appropriate form of separation for the theory of $k$-spaces, but it is based on the presence of a rich supply of closed compact $T_{2}$-subspaces in $X$. In the absence of these, several attempts are known to formulate a useful theory of $k$-spaces; the best known being based on the idea that a topological space $X$ ought to be called compactly generated precisely when its topology agrees with the coinitial topology for all continuous maps $K \rightarrow X$ from compact Hausdorff spaces $K$ into $X$.

The authors gratefully acknowledge support through the National Science Foundation and the Deutsche Forschungsgemeinschaft (in the case of the first) and the Alexander von Humboldt-Stiftung (in the case of the second author).

(c) 1984 Australian Mathematical Society 0263-6115/84 \$A2.00+0.00 
We propose an alternative which is based on recent developments in the theory of locally quasicompact sober spaces in the light of continuous lattices [Gierz, Hofmann, Keimel, Lawson, Mislove, and Scott [2], Hofmann and Mislove [3]] and certain generalizations of their theory.

Firstly, a subset $Q$ of a space $X$ is called quasicompact if and only if it has the Heine-Borel property. A subset $Y$ of $X$ is called saturated, if it agrees with the intersection of its filter of open neighborhoods. A subset $Q$ of $X$ is quasicompact if and only if its saturation $\cap\{U: Q \subseteq U \in O(X)\}$ is quasicompact. The relevant quasicompact sets are, therefore, the saturated ones. We denote their collection with $Q(X)$. The set $Q(X)$ is a semilattice with respect to finite unions; it is not in general closed under the formation of finite intersections. A descending family in $Q(X)$ may very well have an empty intersection, as is illustrated by the example $X=N, O(X)=\{\varnothing\} \cup\{[n, \infty[\}$ and the descending family $O(X) \backslash\{\varnothing\}$. This is in stark contrast with the Hausdorff case. Such pathology, however, vanishes if $X$ is a sober space, that is, a space in which every irreducible closed set has a unique dense point. (A set is irreducible if it cannot be represented as a union of two proper closed subsets.) This was shown by Hofmann and Mislove [3]. It is for this reason that we will be concerned mostly with sober spaces. Another reason, hardly less cogent, is that knowlege of $O(X)$ as a lattice allows the reconstruction of the topological space $X$ precisely if $X$ is sober (see for example [2], Chapter V).

Can the set $Q(X)$ be reconstructed from the lattice $O(X)$ ? The answer is yes: A subset $Q$ of $X$ is quasicompact if and only if its filter $U(Q)$ of open neighborhoods is, as a subset of $O(X)$, open relative to a natural topology defined on complete lattices, namely, the so-called Scott topology. This topology was considered in the context of $O(X)$ by Day and Kelly [6] and is described in detail by Gierz, Hofmann, Keimel, Lawson, Mislove, and Scott [2]. It has become a crucial tool in lattice theoretical discussions which link order and topology. Thus there is a bijection between the set $Q(X)$ and the set OFilt $(O(X))$ of Scott open filters on $O(X)$, and this bijection is order reversing. In order to make this correspondence convenient, we will consider on $Q(X)$ the reverse order, so that $Q(X)$ is in fact an inf-semilattice with largest element $\varnothing$. Since $\operatorname{OFilt}(O(X))$ is completely determined by the lattice structure of $O(X)$, then $Q(X)$ is a lattice invariant of $O(X)$, too.

Now the question is whether indeed complete information on the semilattice $Q(X)$ allows us to reconstruct $O(X)$ (and hence, in the case of sober spaces $X$, the space $X$ itself). This is not always the case as one knows from the example of any Hausdorff space $X$ which is not a $k$-space. A space for which this reconstruction is possible should reasonably be called quasicompactly generated.

In order to find a suitably elegant description of a way to construct $O(X)$ from $Q(X)$ we have to turn to the second point: Duality of partially ordered sets and 
semilattices. We consider the category SL of (inf-) semilattices $L$ with identity which are up-complete in the sense that each directed subset $D$ of $L$ has a sup in $L$ (see Gierz, Hofmann, Keimel, Lawson, Mislove, and Scott [2]). The morphisms in the category are maps $f: L \rightarrow M$ which preserve identities, finite infs and directed sups. (It is shown by Gierz, Hofmann, Keimel, Lawson, Mislove, and Scott [2] that the preservation of directed sups is equivalent to continuity with respect to the Scott topologies.) For an SL-object $L$ the set $\operatorname{SL}(L, 2)$ of morphisms into the two element lattice 2 is itself an SL-object if it is given the structure inherited from $2^{L}$. We call this object the dual of $L$ and write $L^{\wedge}$. For each $L$ there is a natural morphism $h_{L}: L \rightarrow L^{\wedge \wedge}$ given by $h_{L}(x)(c)=c(x)$ for all $c \in L$. The operation `itself is a contravariant self-functor of SL. All of this is a very general formalism which for example is familiar for topological abelian groups. We say that an object $L$ has duality, if $h_{L}: L \rightarrow L^{\wedge \wedge}$ is an isomorphism. The operation with duals is greatly facilitated by an alternative description of $L^{\wedge}$ : If $c \in L^{\wedge}$, then the characteristic function $c_{U}$ of $U$ is a member of $L^{\wedge}$. Thus $L^{\wedge}$ and the semilattice OFilt $L$ of Scott open filters of $L$ are naturally isomorphic. We remark that even for a complete lattice $L$ the dual $L^{\wedge}$ may not be a lattice; it is for this reason that the duality theory which we have to apply to treat the relation of $O(X)$ and $Q(X)$ has to leave the domain of complete lattices and transgress at least into the domain of up-complete semilattices.

In the context of topological spaces we may now reproduce our discussion by saying that the dual $O(X)^{\wedge}$ of $O(X)$ in the category SL is (naturally isomorphic to) $Q(X)$. In particular, we note $O(X)^{\wedge}=Q(X)^{\wedge}$. Hence we have a natural map $j_{X}: O(X) \rightarrow Q(X)^{\wedge}$. If we identify $Q(X)^{\wedge}$ with OFilt $(Q(X))$, then $j_{X}(U)=\{C \in$ $Q(X): C \subseteq U\}$ (where we assume that $X$ is sober; recall that the order on $Q(X)$ is reverse containment). That such a set of quasicompact saturated sets is a Scott open filter was shown by Hofmann and Mislove in [3]. Now it is clear that $O(X)$ and thus $X$ (in the sober case) can be reconstructed from the quasicompact saturated sets of $X$ exactly when $j_{L}$ is an isomorphism, that is, if and only if $O(X)$ has duality in the sense of the category SL. Thus we propose the following general definition:

Definition. A space $X$ is a $q$-space or is quasicompactly generated if and only if it is sober and $O(X)$ has duality (in the sense of the category SL).

Our discussion shows that the definition is reasonable. However, it is certainly desirable to know for sure that sufficiently large classes of spaces which could reasonably be expected to be determined by their quasicompact subsets and that the class of all $k$-spaces both belong to the class of $q$-spaces. In this paper we 
propose to provide the order theoretical background for a proof of the following result:

Proposition. Let $X$ be a sober space such that every quasicompact subset $C$ is contained in some locally quasicompact and properly embedded subspace $Y \subseteq X$ (where a continuous map is proper if the pull-back of any quasicompact saturated set in the range is quasicompact and the lower set (relative to the order given by $x \leqslant y$ if and only if $x \in\{y\}^{-}$) of the image of a closed set is closed), and suppose that a set $U \subseteq X$ is open if and only if its intersection with any properly embedded locally quasicompact subset $Y$ of $X$ is open in $Y$. Then $X$ is a q-space.

As an immediate corollary we obtain the remark

COROLlARY. Every Hausdorff $k$-space is a q-space.

For a proof of these facts we have to develop some tools about the duality of semilattices in SL. Just as in the analogous situation of abelian groups one knows certain well-studied classes of objects with duality such as the class of locally compact abelian groups one has a well understood subclass of objects in SL with duality, namely, the class of continuous semilattices which were first investigated by Lawson (see [5], see also Gierz, Hofmann, Keimel, Lawson, Mislove, and Scott [2], page 191). The numerous ways in which continuous lattices, semilattices, and posets are related to the concept of local quasicompactness have been exhibited by Gierz, Hofmann, Keimel, Lawson, Mislove, and Scott in [2], and the more recent Proceedings of the First Bremen Workshop on Continuous Lattices edited by B. Banaschewski and R.-E. Hoffmann contains further information [1]. Suffice it here to recall that for a sober space $X$ the lattice $O(X)$ is continuous if and only if $X$ is locally quasicompact and that each distributive continuous lattice is (isomorphic to) such an $O(X)$. Since continuous semilattices have duality, this implies in particular that any locally quasicompact sober space is a $q$-space. But the example of a $k$-space $X$ which is not locally compact (such as for instance any first countable space which is not locally compact) yields an example $O(X)$ of a complete Heyting algebra (frame, locale, Brouwerian lattice) with duality which is not continuous.

Similarly to the case of abelian groups one is therefore motivated to seek construction methods which allow us to pass from known objects with duality to more general ones which still satisfy duality. In this regard we will establish the following result: 
TheOREM. Let $L$ be a semilattice in SL and $d_{j}: L \rightarrow L_{j}$ a limit cone of SL-maps into semilattices $L_{j}$ with duality, and suppose that the following two conditions are satisfied:

(A) For each $x \in L$ and each Scott open filter $U$ of $L$ containing $x$, there is an index $j$ and a Scott open filter $U_{j}$ in $L_{j}$ containing $x$ such that $d_{j}^{-1} U_{j} \subseteq U$.

(B) For each $x \in L$ and each Scott open filter $U$ of $L$ not containing $x$ there is an index $j$ such that $\uparrow d_{j} U\left(=\left\{y \in L_{j}\right.\right.$ : there is an $x \in L$ with $\left.\left.d_{j} x \leqslant y\right\}\right)$ is Scott open and does not contain $d_{j} x$.

Then $L$ satisfies duality.

This theorem is one of the following type: If an object $L$ allows "sufficiently many and sufficiently good" test morphisms into objects with duality, then it itself has duality. The proof of the proposition above is reduced to applying the theorem with $L=O(X)$ and $L_{j}=O\left(Y_{j}\right)$ for suitable locally quasicompact spaces $Y_{j}$; the morphisms $d_{j}$ will arise from proper continuous maps $f_{j}: Y_{j} \rightarrow X$.

The following questions arise naturally:

$i$. Is the category of $q$-spaces reflective in the category of sober spaces (in a way similar to the reflective embedding of the category of $k$-spaces into the category of Hausdorff spaces)?

ii. Is the category of $q$-spaces cartesian closed?

We shall address these questions another time.

The paper has benefitted from the referee's careful scrutiny.

\section{Preliminaries in general topology}

Our general references are [1] and [2]; for easy reference we recall at this time some notation and a few relevant results. For an element $a$ in a poset $P$ let $\uparrow a$ denote $\{x \in P: a \leqslant x\}$, and for $A \subseteq P$, set $\uparrow A=\bigcup\{\uparrow a: a \in A\}$. The sets $\downarrow a$ and $\downarrow A$ are defined analogously.

1.1. Definitions. A lattice $L$ is called a complete Heyting algebra (or a frame, local lattice, complete Brouwerien lattice, locale) if and only if it is complete and satisfies the infinite distributive law

$$
x \wedge \vee X=\vee(x \wedge X) \text { for all } x \in L, X \subseteq L .
$$

An element $p$ of an (inf) semilattice $L$ is called prime if and only if $L \backslash \downarrow p$ is a non-empty subsemilattice (that is, if and only if $p \neq 1$ and $x y \leqslant p$ implies $x \leqslant p$ or $y \leqslant p$ ). The space of all prime elements of $L$ carrying the spectral or hull-kernel topology is called the spectrum of $L$ and is written $\operatorname{Spec} L$; here the hull-kernel 
topology is generated by all hulls $\operatorname{Spec} L \cap \uparrow x, x \in L$ as subbasic closed sets; if $L$ is a complete lattice, then the hulls themselves already form the closed sets of the hull-kernel topology.

With a topological space $X$ one associates its topology $O(X)$, the lattice of all open sets under containment. The complement $A$ of a prime element $U \in$ $\operatorname{Spec}(O(X))$ is called irreducible; an irreducible set is characterized by the fact that $A=A_{1} \cup A_{2}$ for closed subsets $A_{j}, j=1,2$, implies $A_{1}=A$ or $A_{2}=A$. Evidently every singleton closure is irreducible; a space is called sober if and only if every closed irreducible set has a unique dense point.

1.2. RemarKs. $i$. The spectrum of a complete Heyting algebra $L$ is a sober space (hence is, in particular, $T_{0}$ ). The function

$$
x \mapsto X \backslash\{x\}^{-}: X \mapsto \operatorname{Spec}(O(X))
$$

is a continuous function which under the pulling back of open sets induces an isomorphism of Heyting algebras from $O(\operatorname{Spec}(O(X)))$ to $O(X)$. The functor $X \mapsto \operatorname{Spec}(O(X))$ is a left reflection from the category of topological spaces onto the full subcategory of sober spaces, called the sobrification.

From the viewpoint of studying topological spaces $X$ and continuous maps via the lattices $O(X)$ and the maps induced between them by continuous maps it is therefore no essential loss to restrict one's attention to sober spaces.

ii. A morphism of complete Heyting algebras is a map $f: L \rightarrow M$ between complete Heyting algebras preserving finite infs and arbitrary sups (as is suggested by the defining identity in 1.1). The category of complete Heyting algebras is denoted HEYT. The function

$$
X \mapsto \operatorname{Spec} L \backslash \uparrow x: L \rightarrow O(\operatorname{Spec} L)
$$

for a complete Heyting algebra $L$ is a morphism in HEYT which is injective (hence an isomorphism, since it is always surjective!) if and only if $L$ is order generated by its primes (that is, every element is an inf of primes). Such complete Heyting algebras are therefore called topologies or complete Heyting algebras with points; their full subcategory is denoted $\mathrm{HEYT}_{0}$. The function $L \mapsto O(\operatorname{Spec} L)$ is a left reflection of HEYT onto HEYT .

From the viewpoint of studying concrete topological spaces via their topologies it is therefore no restriction of generality to concentrate on the subcategory $\mathrm{HEYT}_{0}$, although much attention is given in some quarters to HEYT, its opposite category and the study of topological spaces without points, called locales.

iii. (The Duality Theorem for Spaces and complete Heyting algebras.) The functors Spec and $O$ establish a duality between the categories $\mathrm{HEYT}_{0}$ of complete Heyting algebras with points and the category SOB of sober spaces and continuous maps. 
There is another relation between topology and order to which we must occasionally refer, and which we recall in the following section:

1.3. Definition. i. For a topological space $X$ we set $x \leqslant y$ if and only if $x \in\{y\}$. This defines a quasi-order called the specialisation order. It is a partial order if and only if $X$ satisfies $T_{0}$.

ii. A subset $A \subseteq X$ is called saturated if and only if $A=\cap\{U: A \subseteq U \in O(X)\}$, and for an arbitrary subset $Y$ we call $\cap\{U: Y \subseteq U \in O(X)\}$ its saturation.

In terms of the specialisation order, the closure $\bar{Y}$ of a subset contains the lower set $\downarrow Y$ and is itself a lower set (so that closed sets are lower sets), while the saturation sat $Y$ of $Y$ is the upper set $\uparrow Y$.

iii. A subset $K \subseteq X$ is called quasicompact if and only if for any directed family of open sets $U_{j}$ with $K \subseteq \cup U_{j}$ there is an index $k$ with $K \subseteq U_{k}$. (This is equivalent to the Heine-Borel property which says that every open cover of $K$ allows a finite subcover.)

1.4. Remarks. i. A subset $K$ of $X$ is quasicompact if and only if its saturation sat $K$ is quasicompact.

ii. Finite unions of quasicompact sets are quasicompact.

iii. If $X$ is sober, then for any filtered family $K_{j}$ of quasicompact subsets and any open set $U$ the relation $\cap K_{j} \subseteq U$ implies the existence of an index $k$ such that $K_{k} \subseteq U$. In particular, a filter-basis of quasicompact sets has a non-empty intersection. (See Hofmann and Mislove [3].)

In view of these remarks, it is no loss of generality in discussing quasicompactness to focus one's attention on quasicompact and saturated sets. We will denote the collection of all quasicompact and saturated subsets of $X$ with $Q(X)$ and endow $Q(X)$ with the order of reverse containment, that is, we write $C \leqslant K$ in $Q(X)$ if and only if $K \subseteq C$. Then $Q(X)$ is an inf-semilattice with identity $\varnothing$ in which every directed subset has a supremum. It is a particular property of $X$ that $Q(X)$ is a lattice (see Hofmann and Mislove [3]).

\section{Duality of up-complete semilattices and its application in general topology}

2.1. Definition. i. An up-complete semilattice $L$ is an inf-semilattice with identity in which every directed subset has a sup.

ii. The Scott-topology $\sigma(K)$ on an up-complete semilattice $L$ consists of all subsets $U$ with $U=\uparrow U$ such that the relation $\sup D \in U$ for a directed subset $D \subseteq L$ implies $D \cap U \neq \varnothing$. 
iii. A morphism of up-complete semilattices $f: L \rightarrow M$ is a Scott-continuous semilattice morphism between up-complete semilattices.

iv. The class of up-complete semilattices together with the class of their morphisms form a category SL, the category of up-complete semilattices.

2.2. Remark. A function between up-complete semilattices is a morphism if and only if it preserves identities, finite infs, and directed sups. (See [2], page 112, Proposition 1.2; the equivalence of (1)-(3) is valid for SL-objects.)

Every complete lattice is of course an up-complete semilattice; every morphism of complete Heyting algebras is an SL-morphism. Hence we may write

\section{HEYT $\subseteq$ SL.}

In particular, the lattice 2 of two elements is an SL-object. If $L$ is an SL-object then the hom-set $\operatorname{SL}(L, 2)$ inherits from $2^{L}$ the structure of an up-complete semilattice. (Indeed more generally, if $M$ is a meet-continuous lattice (see, for example, Gierz, Hofmann, Keimel, Lawson, Mislove, and Scott, pages $30 \mathrm{ff}$.), then $\operatorname{SL}(L, M)$ is an SL-object under pointwise operations.)

We denote $\operatorname{SL}(L, 2)$ with its structure of an up-complete semilattice by $L^{\wedge}$ and call it the dual of $L$. For a morphism $f: L \rightarrow M$ in SL we define a morphism $\hat{f}$ : $\hat{M} \rightarrow L^{\wedge}$ as usual by $\hat{f}(c)=c f$. Thus we obtain a contravariant functor of SL into itself, and it is (for the categorist) a routine verification to show that " is adjoint to itself on the right, that is, that there is a natural isomorphism $\operatorname{SL}\left(L, M^{\hat{}}\right) \cong \operatorname{SL}\left(M, L^{\wedge}\right)$. This formalism is familiar from vector spaces or topological abelian groups. A portion of this formalism is the natural map

$$
h_{L}: L \rightarrow L^{\wedge}, \quad h_{L}(x)(c)=c(x) \text { for } x \in L, c \in L .
$$

We say that $L$ has duality or satisfies duality if and only if $h_{L}$ is an isomorphism.

What is different in the category SL from other categories carrying a similar formalism is the fact that the dual $L^{\wedge}$ has a second, more geometrical interpretation which is relevant for our applications and which we discuss next.

2.3. Definition. A subset $U$ of an SL-object $L$ is called an open filter (or more accurately a Scott-open filter) if and only if it is Scott open (that is, $U \in \sigma(L)$ ) and a filter (semilattice and upper set). The totality of open filters will be denoted with OFilt $L$; the containment provides it with a natural partial order relative to which it is a up-complete semilattice.

2.4. Remark. The function $c \mapsto c^{-1}(1)$ provides an isomorphism $L^{\wedge} \rightarrow$ OFilt $L$ whose inverse is given by $U \mapsto c_{U}$ where $c_{U}$ is the characteristic function of $U$. If $f$ : $L \rightarrow M$ is an SL-morphism, then the function $U \mapsto f^{-1} U$ : OFilt $M \rightarrow$ OFilt $L$ is 
an SL-morphism OFilt $f$ which makes the following diagram commutative:

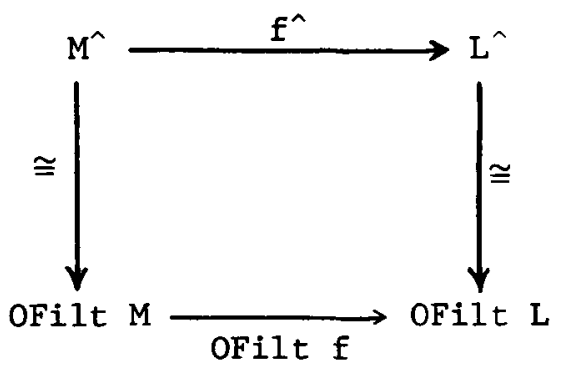

Finally there is a natural SL-morphism $x \mapsto \mathscr{Q}_{L}(x): L \rightarrow$ OFilt(OFilt $\left.(L)\right), \mathscr{Q}_{L}(x)$ $=\{U \in$ OFilt $L: x \in U\}$ such that the following diagram is commutative:

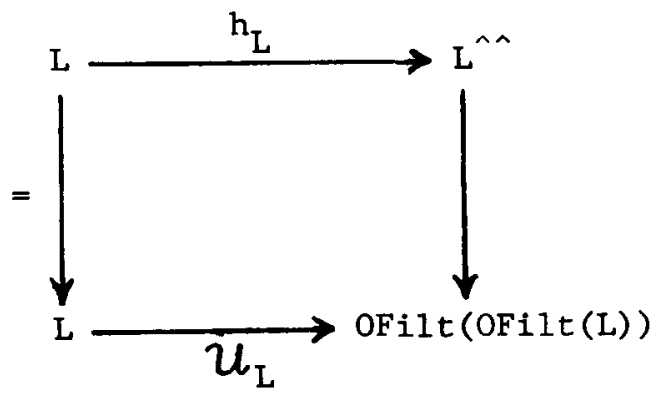

For our purposes it is more convenient to work with open filters than with characters. Hence we make the following convention:

2.5. Convention. We identify $L^{\wedge}$ with OFilt $L$, also $\hat{f}$ with OFilt $f$, and $h_{L}$ with $\mathcal{Q}_{L}$. Thus we consider the semilattice of open filters as the dual of a semilattice.

Now we connect the concept of duality with general topology by identifying the dual of $O(X)$ in SL. We observe first that the dual of a complete Heyting algebra is, in general, not even a lattice. This motivates our consideration of the category $\mathrm{SL}$ which is much larger than the category HEYT, let alone $\mathrm{HEYT}_{0}$.

Suppose now that $K$ is a quasicompact subset of a topological space $X$. Then by 1.3 and the definition of an open filter, the filter $\mathcal{U}(K)$ of open neighborhoods of $K$ is Scott open, hence a member of $O(X)^{\wedge}$. Conversely, if $\mathcal{Q}$ is a Scott open filter on $O(X)$, we define $K=\cap \mathcal{Q}$. Then $K$ is saturated (see 1.3), and if $X$ is sober, then $K$ is also quasicompact and thus is a member of $Q(X)$. Moreover, $\mathcal{Q}$ is the 
filter of open neighborhoods of $K$. This was shown by Hofmann and Mislove in [3]. Thus we can formulate for easy reference:

2.6. Remark. The function $K \mapsto \mathcal{U}(K)$ which associates with a quasicompact saturated set $K$ of a topological sober space $X$ its neighborhood filter is an isomorphism $Q(X) \rightarrow O(X)^{\wedge}$.

Notice that a continuous function $f: X \rightarrow Y$ induces a morphism $O(f)$ of complete Heyting algebras: $O(Y) \rightarrow O(X)$ with $O(f)(V)=f^{-1}(V)$, and an SL-morphism $Q(f): Q(X) \rightarrow Q(Y)$ with $Q(f)(K)=$ sat $f(K)$. Under the isomorphism of 2.6, the map $Q(f)$ corresponds to the dual $O(f)$ via the obvious commutative diagram.

If $U \in O(X)$ and $X$ is sober, then by 1.4.iii the set $\mathscr{K}(U)$ of all quasicompact saturated subsets of $U$ is a Scott open filter of $Q(X)$, that is, $\mathscr{K}(U) \in Q(X)^{\hat{n}}$. Note that we have $U=\cup \mathscr{K}(U)$ for any open set $U$. It is readily verified that the following diagram is commutative:



Thus we have

2.7. RemarK. For a sober space $X$, the complete Heyting algebra $O(X)$ satisfies duality in SL if and only if every Scott open filter in $Q(X)$ is of the form $\mathscr{K}(U)$ for an open set $U$ in $X$.

We now formally define the concept of a $q$-space:

2.8. Definition. A topological space $X$ is called a $q$-space or quasicompactly generated space if and only if $O(X)$ satisfies duality, and $X$ is sober.

This may be made explicit as follows; the virtue of the definition of course is in its simplicity and formal elegance made possible through the use of duality. 
2.9. Proposition. For a sober space $X$ the following definitions are equivalent:

(1) $X$ is a q-space.

(2) For each collection $\mathcal{K}$ of quasicompact saturated subsets of $X$, the following statement holds:

Suppose that $\mathscr{K}$ is closed under finite unions and the passage to quasicompact and saturated subsets, and suppose that for every filterbasis $\mathcal{F}$ of quasicompact saturated sets the relation $\cap \mathcal{F} \in \mathcal{K}$ implies $\mathscr{F} \cap \mathscr{K} \neq \varnothing$. Then $\cup \mathscr{K}$ is open and every quasicompact saturated subset of $\cup \mathscr{K}$ is an element of $\mathscr{K}$.

Recall at this point that for a $q$-space $X$ the semilattice $Q(X)$ of quasicompact and saturated sets in $X$ completely determines $O(X) \cong O(X)^{\wedge \wedge}$ as $Q(X)^{\wedge}$; and since $X$ is sober, $X$ can be retrieved as $\operatorname{Spec} O(X)$ from $O(X)$. Thus $Q(X)$ determines $X$. We will exhibit various types of spaces as $q$-spaces.

\section{Galois connections, duality, and proper maps}

Gierz, Hofmann, Keimel, Lawson, Mislove, and Scott have shown in [2] how important the concept of Galois connection is in the study of complete lattices. We utilize this concept in SL. But first we recall the definition:

3.1. Definition. A Galois connection between two posets $S$ and $T$ is a pair of maps $d: S \rightarrow T$ and $g: T \rightarrow S$ such that for all $s \in S$ and $t \in T$ the relations $d s \leqslant t$ and $s \leqslant g t$ are equivalent. The map $d$ is called the lower adjoint and the map $g$ the upper adjoint. We will simply write $(d, g): S \rightarrow T$ when a Galois connection is given.

It is well known that a function between complete lattices which preserves arbitrary sups has an upper adjoint and that every function with an upper adjoint preserves arbitrary sups. The opposite statement holds for the preservation of infs and lower adjoints (see Gierz, Hofmann, Keimel, Lawson, Mislove, and Scott [2], pages $18 \mathrm{ff}$.). In general, SL-morphisms need not have adjoints. However, the following observation illustrates the significance of Galois connections in the context of duality:

3.2. Proposition. Suppose that $d: L \rightarrow M$ and $g: M \rightarrow L$ are two SL-morphisms such that $(d, g): L \rightarrow M$ is a Galois connection. Then $\left(g^{\hat{\prime}}, \hat{d^{\wedge}}\right): M^{\hat{*}} \rightarrow L^{\hat{\prime}}$ is a Galois connection. (Notice that $\mathrm{g}^{\hat{}}$ is now the lower adjoint and $\hat{d}$ is the upper adjoint!) 
Proof. Let $V \in M^{\hat{M}}$ and $U \in L^{\wedge}$. Then $g^{\hat{g}}(V) \leqslant U$ means $g^{-1} V \subseteq U$. But $g^{-1} V=\uparrow d V$ by the definition of Galois adjunctions. But since $U$ is an upper set, the relation $\uparrow d V \subseteq U$ is equivalent to the containment $d V \subseteq U$, which means precisely $V \leqslant d^{-1} U=\hat{d}(U)$.

If $f: X \rightarrow Y$ is a continuous map between spaces, then the morphism $O(f)$ : $O(Y) \rightarrow O(X)$ always has an upper adjoint $G(f): O(X) \rightarrow O(Y)$ given by $G(f)(U)=\cup\left\{V \in O(Y): f^{-1} V \subseteq U\right\}$. This map preserves arbitrary infs, but is not always an SL-morphism, since it may very well fail to be Scott continuous. We now exhibit an important class of functions $f$ for which $G(f)$ is Scott continuous and hence an SL-morphism:

3.3. Proposition. For a continuous function $f: X \rightarrow Y$ between sober spaces the following conditions are equivalent:

(1) (i) The set $f^{-1}(K)$ is quasicompact for each saturated quasicompact set $K$ of $Y$, and

(ii) the set $\downarrow f(C)=\left\{y \in Y: y \in\{f(c)\}^{-}\right.$for some $\left.c \in C\right\}$ is closed for each closed subset $C$ of $X$.

(2) The morphism $G(f): O(X) \rightarrow O(Y)$ is Scott continuous (that is, an SL-morphism).

(3) For each open filter $\mathcal{T}$ in $O(Y)$ the set $G(f)^{-1}(\mathfrak{V})=\uparrow O(f)(\mathcal{V})$ is an open filter in $O(X)$.

Proof. (1) $\Rightarrow(3)$ : Let $\mathcal{T}$ be an open filter in $O(Y)$. Then there is a quasicompact saturated set $K \in Q(Y)$ with $\mathscr{V}=\mathscr{Q}(K)$ by 2.6. Let $\mathscr{Q}=\mathscr{U}\left(f^{-1} K\right)$. By (1)(i) and 2.6, this is an open filter on $O(X)$. We will show that $G(f)^{-1}(\mathcal{V})=Q$ and thereby establish (3). If $U \in \mathfrak{V}$, then $K \subseteq U$, whence $f^{-1} K \subseteq f^{-1} U$ and thus $O(f)(U)=f^{-1} U \in \mathcal{Q}$, and thus $G(f)^{-1}(\mathfrak{V})=\uparrow O(f)(\mathcal{V}) \subseteq \mathcal{Q}$. Conversely, let $V \in \mathcal{Q}$. Then $C=X \backslash V$ is a closed set which is disjoint from $f^{-1} K$. Hence $f(C) \cap K=\varnothing$, and since $K$ is saturated, $\downarrow f(C) \cap K=\varnothing$. But $\downarrow f(C)$ is closed by (1)(ii), hence $U=Y \backslash \downarrow f(C)$ is open. But $K \subseteq U$ and $f^{-1} U \subseteq V$, whence $V \in \uparrow O(f)(\mathbb{V})$. This proves the claim.

(3) $\Rightarrow(2)$ : Let $V_{j}$ be a directed family of open sets in $X$ with union $V$. We denote the union of the $G(f)\left(V_{j}\right)$ with $U$. Since $G(f)$ is order preserving, we know $U \subseteq G(f)(V)$. Now let us assume $U \neq G(f)(V)$. Then we find a $y \in$ $G(f)(V) \backslash U$. Now the filter $\mathcal{Q}(y)$ of open neighborhoods of $y$ is open by 2.6 . Hence by (3) we know that the filter $\mathcal{Q}=\uparrow O(f)($ Q $(y))$ generated by all $f^{-1} W$ with $y \in W$ is open in $O(X)$. Since $y \in G(f)(V)$ we know that $O(f) G(f)(V)=$ $f^{-1} G(f)(V) \in \mathscr{Q}$. But $O(f) G(f)(V) \subseteq V$ since $O(f)$ is a lower adjoint of $G(f)$. Hence $\cup V_{j}=V \in \mathcal{Q}$. The openness of $\mathscr{Q}$ implies the existence of a $j$ with 
$V_{j} \in \mathcal{Q}$. But then $G(f)\left(V_{j}\right) \in \mathcal{U}(y)$, that is, $y \in G(f)\left(V_{j}\right) \subseteq U$, and this is a contradiction.

$(2) \Rightarrow(1)$ : To show (i), let $K$ be a quasicompact saturated subset of $Y$. Then $\mathcal{Q}(K)$ is open in $O(Y)$ by 2.6. Since $G(f)$ is Scott continuous, the filter $G(f)^{-1} \mathscr{Q}(K)=\uparrow O(f)(\mathcal{Q}(K))$ is open, too. Its intersection however is $f^{-1}(K)$. Hence $f^{-1}(K)$ is quasicompact by 2.6. In order to show (ii) we let $C$ be a closed subset of $X$; define $U \in O(X)$ by $U=X \backslash C$. Now let $y \in f(C)^{-}$. Then $y \notin$ $G(f)(U)$, since $G(f)(U)$ is the largest open set $V$ in $Y$ with $f^{-1} V \subseteq U=X \backslash C$. If we denote with $Q$ the prime $Y \backslash\{y\}^{-}$of $O(Y)$ we have $G(f)(U) \subseteq Q$. Then by a lemma on primes by Hofmann and Watkins (see [4], 1.3) there is a prime $P \in O(X)$ with $G(f)(P) \subseteq Q$ and $U \subseteq P$, since $G(f)$ is Scott continuous by (2). Now $P=X \backslash\{x\}^{-}$with $x \in C$ since $X$ is sober, and $G(f)\left(X \backslash\{x\}^{-}\right)=Y \backslash\{f(x)\}^{-}$. The relation $G(f)(P) \subseteq Q$ then implies $\{y\}^{-} \subseteq\{f(x)\}^{-}$, that is, $y \leqslant f(x)$ in the specialisation order of $Y$. Thus $y \in \downarrow f(C)$. This shows that $f(C)^{-} \subseteq \downarrow f(C)$; since always $\downarrow f(C) \subseteq f(C)^{-}$, we deduce that $\downarrow f(C)=f(C)^{-}$is closed.

3.4. Definition. A function between two sober spaces $f: X \rightarrow Y$ is called proper if and only if it satisfies the three equivalent conditions of Proposition 3.3.

From this Proposition and 1.2.iii we obtain the following corollary:

3.5. CoRollary. The category of sober spaces and proper maps is dual to the subcategory of $\mathrm{Heyt}_{0}$ consisting of all complete Heyting algebras with points and all morphisms whose upper adjoint is Scott continuous (preserves directs sups).

If $f: X \rightarrow Y$ is a proper map, we have the Galois connection $(O(f), G(f))$ : $O(Y) \rightarrow O(X)$. Hence according to 3.1 we also obtain a Galois connection $\left(G(f)^{\wedge}, O(f)^{\wedge}\right): O(Y)^{\wedge} \rightarrow O(X)^{\wedge}$. Via 2.6 this corresponds to a Galois connection $(P(f), Q(f)): Q(Y) \rightarrow Q(X)$ with $P(f)(K)=f^{-1}(K)$ for $K \in Q(Y)$ (and $Q(f)(K)=$ sat $f(K)$ for $K \in Q(X))$.

\section{On the construction of semilattices with duality}

We observe several criteria expressing the property of being dual for an SL-object in terms of other SL-objects which are known to have duality.

4.1. Proposition. Let $L$ be an up-complete semilattice. Then the following conditions are equivalent:

(1) There is a surjective SL-morphism $g: M \rightarrow L$ with a left inverse in SL such that $M$ satisfies duality, that is, $L$ is a retract in SL of an object with duality.

(2) L satisfies duality. 
Proof. (2) $\Rightarrow(1)$ is trivial with $g=1_{L}$.

(1) $\Rightarrow$ (2): Suppose that $\mathscr{Q} \in L^{\wedge}$. We let $d: L \rightarrow M$ be the left inverse of $g$ and define $\mathfrak{V}=\left\{U \in M^{\wedge}: d^{-1} U \in \mathcal{Q}\right\}=d^{\wedge} \wedge(\mathcal{Q})$. Then $\mathfrak{V} \in M^{\wedge}$ and since $M$ has duality there is an $m \in M$ with $\mathcal{V}=\mathscr{Q}(m)$. We claim that $\mathscr{Q}=\mathcal{Q}(g(m))$, which will finish the proof. But $U \in \mathcal{Q}$ if and only if $d^{-1} g^{-1} U=(g d)^{-1} U=U \in \mathscr{Q}$ if and only if $g^{-1} U \in \mathbb{V}$ if and only if $m \in g^{-1} U$ if and only if $g(m) \in U$.

The principal result in this line is a theorem of the following type which we will prove next: If an SL-object $L$ is a (suitable) limit of SL-objects with duality, then $L$ has duality. In order to discuss this situation more accurately, we recall the concept of a limit. Let $J$ be a category and $F: J \rightarrow$ SL a functor. An object $L$ is called a limit of $F$, written $\lim F$, provided that there are natural morphisms $p_{j}$ : $L \rightarrow F(j)$ (one for each object $j$ in $J$; and natural means that for each morphism $f: i \rightarrow j$ in $J$ one has $\left.p_{j}=F(f) p_{i}\right)$ such that for any system $q_{j}: E \rightarrow F(j)$ of natural morphisms there is a unique solution $q: E \rightarrow L$ such that $q_{j}=p_{j} q$ for all $j$. If $J$ is a set (that is, a small category) then one can identify the limit $L$ with the subset in the product $\Pi_{j \in \mathrm{ob} J} F(j)$ of all $\left(x_{j}\right)_{j \in \mathrm{ob} J}$ with $F(f)\left(x_{\operatorname{dom} f}\right)=x_{\operatorname{ran} f}$ for all $f \in \operatorname{morph} J$ (where dom $f$ is the domain of $f$ and $\operatorname{ran} f$ its range). The natural maps $p_{j}$ are then just the restrictions of the projections. We record from this the following remark:

4.2. LEMMA. If $L=\lim F$ and if we find for each $j$ an element $x_{j} \in F(j)$ in such a fashion that $F(f)\left(x_{\operatorname{dom} f}\right)=x_{\operatorname{ran} f}$, then there is a unique element $x \in L$ with $p_{j}(x)=x_{j}$.

4.3. Theorem. Let $L$ be an up-complete semilattice. Then the following conditions are equivalent:

(1) $L=\lim F$ for some functor such that the following conditions are satisfied:

(i) $F(j)$ satisfies duality for all $j$.

(ii) For each $x \in L$ and each open filter $U \in L^{\wedge}$ with $x \in U$ there is a $j$ and an open filter $V$ in $F(j)$ containing $p_{j}(x)$ such that $p_{j}^{-1}(V) \subseteq U$.

(iii) For each $x \in L$ and each open filter $U \in L^{\wedge}$ with $x \notin U$ there is a $j$ and an upper adjoint $g_{j}: F(j) \rightarrow L$ of $p_{j}$ in SL such that $p_{j}(x) \notin p_{j}(U)$, that is, $g_{j}\left(p_{j}(x)\right) \notin U$.

(2) L satisfies duality.

Proof. (2) $\Rightarrow(1)$ is trivial with the constant functor with value $L$.

$(1) \Rightarrow(2)$ : Let $\mathscr{Q} \in L^{\wedge}$. We must find an $x \in L$ such that $\mathscr{Q}=\mathscr{U}(x)$. For each $j$ we have a map ${p_{j}^{\wedge}}^{\wedge}: L^{\wedge} \rightarrow F(j)^{\wedge}$. In particular, $p^{\wedge \wedge}(U)$ is an open filter on $F(j) \hat{~}$. In fact,

(a) $p_{j}^{\wedge}($ Q $)=\left\{V \in F(j) \hat{}: p_{j}^{-1}(V) \in \mathcal{Q}\right\}$. 
Since $F(j)$ satisfies duality, there is a unique element $x_{j} \in F(j)$ such that (b) $p_{j} \hat{\wedge}(\mathcal{Q})=\mathscr{Q}\left(x_{j}\right)$.

From (a) and (b) we conclude:

(c) For an open filter $V$ on $F(j)$ one has $x_{j} \in V$ if and only if $p_{j}^{-1}(V) \in \mathcal{Q}$.

For any morphism $f$ in the domain category $J$ of $F$ we have a commutative diagram

(d)

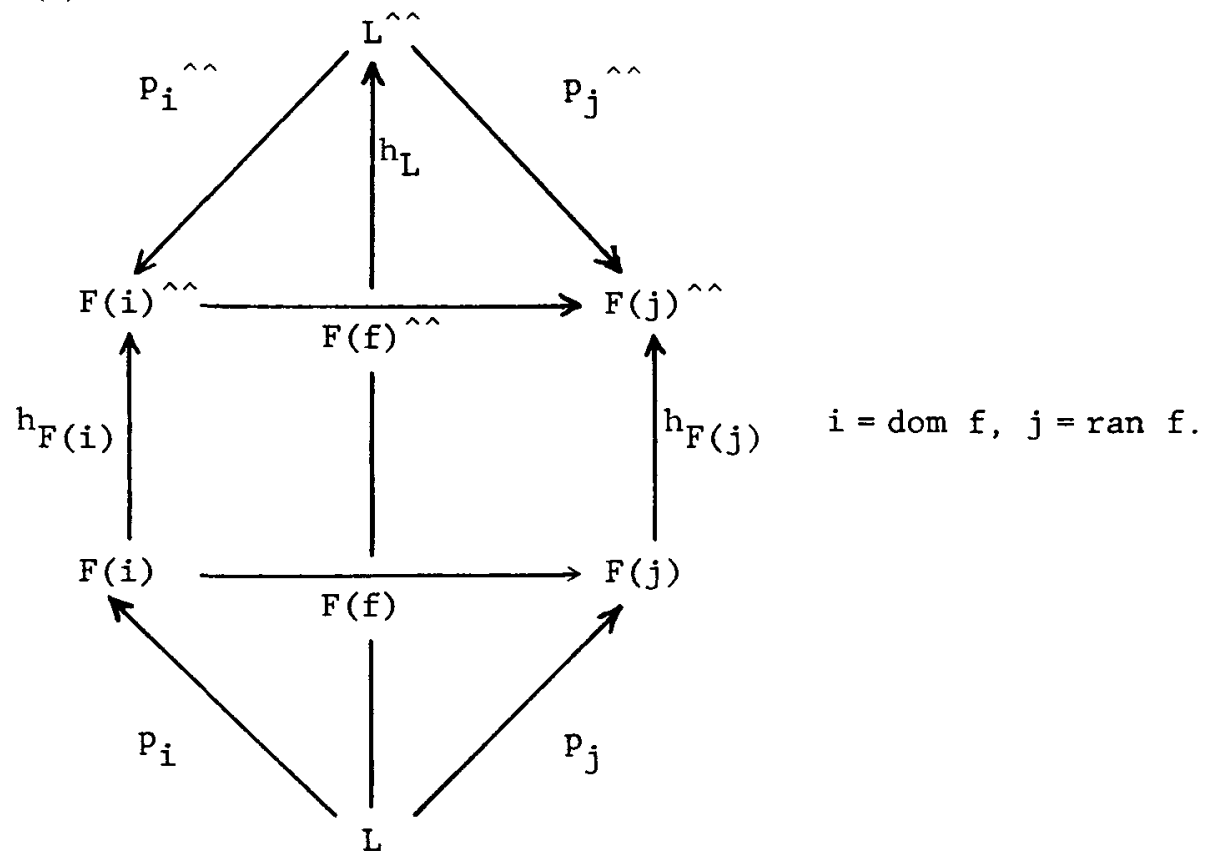

From this diagram we deduce

(e) $F(f)\left(x_{i}\right)=x_{j}$ for all $f: i \rightarrow j$ in $J$.

By Lemma 4.2 we obtain an element $x \in L$ uniquely such that

(f) $p_{j}(x)=x_{j}$ for all $j$ in $J$.

Now we claim that $\mathscr{U}=\mathscr{U}(x)$.

Firstly, let $U \in \mathscr{Q}(x)$. Then $x \in U$. Hence by condition (ii) there is a $j$ in $J$ and an open filter $U_{j}$ in $F(j)$ with $x_{j}=p_{j}(x) \in U_{j}$ and $p_{j}^{-1}\left(U_{j}\right) \subseteq U$. But by (c) the relation $x_{j} \in U_{j}$ implies $p_{j}^{-1}\left(U_{j}\right) \in \mathcal{Q}$, whence $U \in \mathcal{Q}$.

Secondly, suppose that $U \in \mathcal{Q}$. We claim $x \in U$. Assume not. Then by condition (iii) there is a $j$ in $J$ such that $x_{j}=p_{j}(x) \notin \uparrow p_{j}(U)$. Since $p_{j}$ has an upper adjoint, $\uparrow p_{j}(U)=g_{j}^{-1}(U)$ is an open filter. Since $U \subseteq p_{j}^{-1}\left(\uparrow p_{j}(U)\right)$ and $U \in \mathcal{Q}$ we have $p_{j}^{-1}\left(\uparrow p_{j}(U)\right) \in \mathcal{Q}$. Then by (c) we have $x_{j} \in \uparrow p_{j}(U)$. But this is a contradiction. The proof is complete. 
Notice that we did use the hypothesis in (iii) that the upper adjoint $g_{j}$ is in SL, that is, preserves directed sups. It was this property which allowed us to conclude that $\uparrow p_{j}(U)=g_{j}^{-1}(U)$ was open. It would, however, have sufficed in condition (iii) to demand instead of the existence of the upper adjoint $g_{j}$ that $\uparrow d_{j}(U)$ be open. To understand condition (iii) fully, recall that $g_{j}\left(p_{j}(x)\right) \geqslant x$, so that, in principle, $x \notin U$ does not imply $g_{j}\left(p_{j}(x)\right) \notin U$.

Condition (ii) in 4.3(1) has an equivalent formulation in terms of topologies. For this purpose, we introduce in an up-complete semilattice, in addition to the Scott topology $\sigma(L)$, another possibly coarser topology.

4.4. Definition. Let $L$ be an up-complete semilattice. The set OFilt $L$ of Scott-open filters of $L$ is an intersection closed basis for a topology $\varphi(L)$, called the filter topology. The topological space $(L, \varphi(L))$ we simply denote $\Phi L$.

Since $\varphi(L) \subseteq \sigma(L)$, every $\varphi(L)$-open filter is in OFilt $L$ and by definition, OFilt $L \subseteq \varphi(L)$. Hence

4.5. RemarK. For an up-complete semilatice, the semilattice $L^{\wedge}=$ OFilt $L$ is also the semilattice of $\varphi(L)$-open filters.

Now we can reformulate condition 4.3(1)(ii):

4.6. Proposition. In Theorem 4.3, condition (1)(ii) is equivalent to the following condition

(ii') $\Phi(\lim F)=\lim \Phi F$.

In other words: The filter topology of the semilattice $L$ is the limit of the spaces $F(j)$ with their filter topology in the category of topological spaces.

Proof. It is clear that any SL-morphism is continuous relative to the filter topologies, so that $\Phi$ is a functor from SL into the category of topological spaces and continuous maps. In particular $\Phi F$ is a functor into the category of topological spaces. Limits are calculated in this category just as we described it for SL. Thus the underlying set of $\lim \Phi F$ is that of $L=\lim F$. Its topology is the coarsest making all limit maps $p_{j}$ continuous. Since $p_{j}: \Phi L \rightarrow \Phi_{j} L$ is always continuous, the limit topology is always contained in the filter topology $\phi(L)$. But condition 4.3(1)(ii) exactly ensures the reverse containment.

We now return to topological spaces and use Theorem 4.3 to show that $k$-spaces and indeed a much larger class of non-separated spaces are $q$-spaces. 
The following definition allows a simplification of terminology in the discussion which we are about to undertake.

4.7. Definition. We say that a family $\mathscr{F}$ of functions $f: Y \rightarrow X$ of sober spaces is sufficient (for $X$ ) if and only if the following conditions are satisfied:

(i) Each $f: Y \rightarrow X$ in $\mathcal{F}$ is proper.

(ii) For each $K \in Q(X)$ there is an $f \in \mathscr{F}$ with $K \subseteq$ im $f$.

(iii) If $U_{f}$ is open in $Y$ for $f: Y \rightarrow X$ in $\mathscr{F}$ such that the family $\left\{U_{f}: f \in \mathscr{F}\right\}$ satisfies $g^{-1}\left(U_{f^{\prime}}\right)=U_{f}$ for every continuous function $g: Y \rightarrow Y^{\prime}$ with $f^{\prime}=f g$ for $f, f^{\prime} \in \mathscr{F}$, then there is an open set $U$ in $X$ such that $U_{f}=f^{-1}(U)$.

4.8. THEOREM. For a sober space, the following conditions are equivalent:

(1) There is a sufficient family $\mathscr{F}$ of maps $f: Y \rightarrow X$ such that all $Y$ are $q$-spaces.

(2) $X$ is a q-space.

Proof. (2) $\Rightarrow(1)$ is trivial with $\mathscr{F}=\left\{1_{X}\right\}$.

(1) $\Rightarrow(2)$ : The maps $f \in \mathscr{F}$ are the objects of a category $J$ whose morphism $f_{1} \rightarrow f_{2}$ are given by continuous proper maps $g: Y_{2} \rightarrow Y_{1}$ such that the diagram

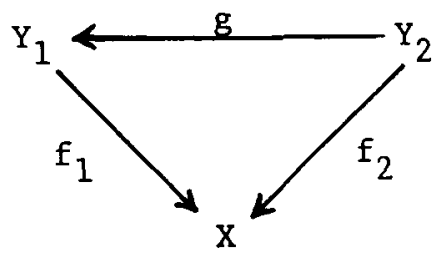

is commutative. For each $f: Y \rightarrow X$ in $\mathscr{F}=o b J$, the topology $O(Y)$ is in particular an up-complete semilattice, so that we have a functor $O: J \rightarrow$ SL which associates with a morphism $f_{1} \rightarrow f_{2}$ the SL-map $O(g): O\left(Y_{1}\right) \rightarrow O\left(Y_{2}\right)$. We set $L=O(X)$ and obtain for each $f \in \mathscr{F}$ an SL-morphism $p_{f}=O(f): L \rightarrow O(Y)$. The map $g_{f}: O(Y) \rightarrow L$ is given by $g_{f}=G(f)$ and is the upper adjoint of $p_{f}$. By Proposition 3.3, it is an SL-morphism.

Condition 4.7(iii) implies that $L=\lim O$. In order to complete the proof, it now suffices to verify conditions (ii) and (iii) of $4.3(\mathrm{i})$; then Theorem 4.3 will establish the claim, since condition 4.3(1)(i) is satisfied by 4.8(1).

Ad 4.3(1)(ii): Let $U$ be an open set in $X$ and $Q$ a Scott open filter on $O(X)$ containing $U$. Then by 2.6 , the set $K=\cap \mathscr{U}$ is quasicompact and saturated. By 4.7(ii) there is an $f \in$ ob $J$ with $K \subseteq \operatorname{im} f$. The filter $थ\left(f^{-1} K\right)$ on $Y=\operatorname{dom} f$ of 
open neighborhoods of the quasicompact set $f^{-1} K$ in $Y$ is Scott open in $O(Y)$ by 2.6, and it contains $f^{-1} U=p_{f}(U)$. Furthermore, an open set $W$ of $X$ is in $p_{f}^{-1}\left(\mathscr{Q}\left(f^{-1} K\right)\right)$ if and only if $f^{-1} W=p_{f}(W) \in \mathscr{Q}\left(f^{-1} K\right)$ if and only if $f^{-1} K \subseteq$ $f^{-1} W$, and this implies $K=f f^{-1} K$ (since $\left.K \subseteq \operatorname{im} f !\right) \subseteq f f^{-1} W \subseteq W$, that is, $W \in \mathcal{Q}(K)$. Thus 4.3(1)(ii) is proved.

Ad 4.3(1)(iii): Let $U$ be an open set of $X$ and $U$ a Scott open filter on $O(X)$ not containing $U$. If $K=\cap \mathcal{Q}$, then $K \in Q(X)$ and $K \not Z U$. By 4.7(ii) we find an $f \in \mathrm{ob} J$ with $K \subseteq \operatorname{im} f$. Assume that $p_{f}(U) \in \uparrow p_{f}(\mathcal{Q})$; then there is an open set $W$ of $X$ such that $f^{-1} W=p_{f}(W) \subseteq p_{f}(U)=f^{-1} U$ and $K \subseteq W$. Then $K=f f^{-1} K$ (since $K \subseteq \operatorname{im} f !) \subseteq f f^{-1} U \subseteq U$, and this is a contradiction. Thus 4.3(1)(iii) is proved and the theorem established.

We recall that any locally quasicompact sober space is a $q$-space: indeed if $Y$ is locally quasicompact, then $O(Y)$ is a continuous lattice (see [2], page 259). But then $O(Y)$ has duality ([2], page 191). Thus $Y$ is a $q$-space, if in addition it is sober (2.8). The following corollary then is a generalisation of this fact:

4.9. CoRollary. If a sober space $K$ allows a sufficient family of maps $f: Y \rightarrow X$ with $Y$ locally quasicompact (and sober), then $X$ is a q-space.

Proof. This is immediate from Theorem 4.8 and the preceding remarks.

4.10. COROLlaRY. Suppose that $X$ is a sober space with the following properties:

(i) Every quasicompact saturated subset is locally quasicompact, sober, and closed in $X$.

(ii) A subset $U$ of $X$ is open if and only if $U \cap K$ is open for every quasicompact saturated subset $K$ of $X$.

Then $X$ is a q-space.

PROoF. If every quasicompact saturated subset is closed, then every quasicompact saturated subset is properly embedded into $X$. Then by (i) and (ii) the family of inclusion maps of the subspaces $K \in Q(X)$ is sufficient for $X$. The assertion then follows from 4.9 .

4.11. COROLlaRY. Every $t_{2} k$-space is a $q$-space.

4.12. COROLlaRY. Every Hausdorff $k$-space is a $q$-space. 


\section{References}

[1] B. Banaschewski and R. E. Hoffmann, editors, Continuous lattices, Proceedings of the Conference held at Bremen 1979 (Lecture Notes in Mathematics 871, Springer-Verlag, Berlin, Heidelberg, and New York, 1981).

[2] G. Gierz, K. H. Hofmann, K. Keimel, J. D. Lawson, M. Mislove, and D. Scott, A compendium of continuous lattices (Springer-Verlag, Berlin, Heidelberg and New York, 1980).

[3] K. H. Hofmann and M. Mislove, 'Local compactness and continuous lattices,' in [1] above, pp. 209-248.

[4] K. H. Hofmann and F. Watkins, 'The spectrum as a functor,' in [1] above, pp. 249-263.

[5] J. D. Lawson, 'The duality of continuous posets,' Houston J. Math. 5 (1981), 357-394.

[6] B. J. Day and G. M. Kelly, 'On topological quotient maps preserved by pull-backs of products,' Proc. Cambridge Philos. Soc. 67 (1970), 553-558.

Department of Mathematics

Tulane University

New Orleans, Louisiana 70018

U.S.A.

Fachbereich Mathematik

THD

D 6100 Darmstadt

FR Germany

\author{
Department of Mathematics \\ Louisiana State University \\ Baton Rouge, Louisiana 70803 \\ U.S.A.
}

